\title{
Transgenic trees resistant to microbial pests
}

\author{
by Armand Séguin ${ }^{1}$
}

\section{Introduction}

Each year important losses in forest productivity are suffered due to susceptibility of trees to disease. For instance, important forest trees in Canada like poplar and white pine are seriously affected by fungal pathogens. For the genus Populus (poplar), melampsora leaf rust and septoria leaf spot represent serious disease threats that could impair the full exploitation of intensively-managed plantations for short-rotation cultures. In the case of Pinus strobus (eastern white pine), blister rust has devastated large populations in North America and foresters are still reluctant to use this tree species. Recent progress in plant genetic engineering is spectacular and genetically modified crops are now commercialized. Obviously, a major goal for plant biotechnology is the genetic engineering of disease- and insect-resistant varieties. However, these technologies are not easily applied to forest tree species and some methodologies had required improvements and modifications. Our laboratory at the Canadian Forest Service has a long-term commitment to the development of DNA transformation technologies for trees. This effort has recently translated into routine procedures and highly skilled personnel for the production and analysis of genetically transformed (transgenic) trees. In fact, we were the first laboratory to adapt a DNA transformation protocol for pine based on Agrobacterium tumefaciens. A short description of the DNA transformation procedure used in our laboratory for genetic engineering of trees is presented below.

\section{Tree Defense Response}

The defense system of plants is not as complex as with humans, but includes a variety of mechanisms based on specific gene induction. Plants had developed strategies to recognize specifically various pathogens and initiate various defense reactions. Production of antibiotic molecules (phytoalexins) and enzymes (chitinases) and specific physical modification of the cell wall for limiting the proliferation of the microbial invader are examples of these defense strategies. More recently, it was possible to identify coding sequences for small peptides with in vitro antimicrobial activities. These molecules do not recognize specific components of an invading pathogen like antibodies, but have a wider action spectrum. These anti-microbial peptides (AMPs), as products of single genes, can be synthesized rapidly in a flexible way and because of their small size require low input of energy. In brief, investigation at the cellular level of the molecular mechanisms underlying the natural tree defense response could eventually provide alternatives to the application of chemical fungicides. Also, the identification of some key players (genes) at the genetic level could provide markers essential for facilitating the development of pathogen resistance through more traditional genetic improvement.

\footnotetext{
${ }^{1}$ Canadian Forest Service, Laurentian Forestry Centre, Natural Resources Canada, 1055 du P.E.P.S., P.O. Box 3800, Ste-Foy, Quebec G1V 4C7. E-mail: seguin@NRCan.gc.ca
}

\section{How AMPs Work}

AMPs have recently been identified from a wide variety of organisms like plants, animals and microbes. Well-described examples of AMPs are cecropins that accumulate in the hemolymph of many invertebrates in riposte to injury or infection and the magainins produced by glands of amphibians' skin. AMPs are divided in different categories (linear or disulfide-linked peptides, for example). These small peptides are effective in vitro to kill susceptible bacteria and/or fungi at concentrations of $\mu \mathrm{g}$ 's per liter.

The potential importance of AMPs in plant disease resistance has been acknowledged only recently. The endogenous plant AMPs are commonly cationic amphipathic molecules composed of 12 to 45 amino acid residues (frequently cysteinerich). For example, defensins, thionins, heveins and knottins fall into the general category of plant AMPs, with multiple structures found in various plant species. Interestingly, these peptides can either be produced constitutively in specific tissues or induced after pathogen or stress induction. To date, the only AMPs identified in forest trees species are thionin types from Norway spruce and white spruce. Their deduced protein sequences from complementary DNA showed conservation of specific groups of amino acids between the AMPs of several plants.

Concerning AMPs' mode of action, there are several indications that these peptides play a role at the cytoplasmic membrane forming ions channels. The presence of these channels often results in the destruction of the targeted cell. Our research program using AMPs for engineering trees for improved pest resistance focus essentially on two different AMPs provided by Demeter BioTechnologies, Ltd. The structure of these AMPs is completely synthetic but exploits the fact that natural AMPs have a cationic nature and predisposition toward an amphipathic secondary structure upon interaction with a membrane surface. Other AMPs (cecropin B and magainin II) were included in the in vitro tests together with the peptides provide by Demeter. The peptides were first tested for their inhibition of fungal spore germination from various forest tree pathogens including white pine blister rust and poplar leaf spot and canker. This work, carried out by a team at the Laurentian Forestry Centre led by Dr. Richard Hamelin, showed that all peptides inhibit spore germination but that the two Demeter synthetic peptides showed the strongest antifungal activity. The results from that study were very promising and convinced us to go ahead with the corresponding geneencoding for the AMPs provided by Demeter in the context of tree genetic engineering. However, phytotoxicity of the AMPs for poplar and pine had to be tested first and this was done by pollen germination tests using serial dilutions of the peptide stock solutions. The data obtained were encouraging and demonstrated that inhibition of pollen germination was only observed at high peptide concentrations.

\section{Tree Genetic Transformation}

There are two basic steps in genetic transformation. First, the DNA being introduced has to reach the nucleus of the cell 
that is to be transformed. This foreign DNA will not replace part of an existing chromosome, but will instead be randomly inserted into a chromosomal region. In the vast majority of cases, the introduction of a new gene will not affect the plant's normal genetic expression. Second, the introduced DNA may either be digested by the cell or be integrated into the plant's chromosomes. In the latter case, stable genetic transformation occurs, and the introduced DNA is transmitted to subsequent generations during gamete formation. However, genetic transformation is not achieved after only introducing a particular DNA into the cell nucleus. The transformed cell has to be regenerated in an entire tree. This is where in vitro culture comes into the picture. This technique involves propagating plant tissues (or single cells) in a controlled environment free of microorganisms. Organogenesis, the preferred method in the case of poplar trees, consists of regenerating the entire plant using organs derived from tissue or from isolated cells. For conifers, somatic embryogenesis is the most reliable method and is somehow similar to zygotic embryogenesis observed in normal seed development. These in vitro techniques are also employed in the vegetative propagation of elite trees.

\section{Tree Genetic Engineering with AMP-encoding Genes}

As mentioned earlier, technologies related to genetic transformation of trees have evolved greatly over the last few years. For conifers this technology was essentially based on particle bombardment, consisting of coating microscopic gold particles with DNA for subsequent introduction using an acceleration force. This methodology was used successfully to produce numerous genetically transformed (transgenic) tree lines with various genes including AMPs. More recently, we developed an alternative method based on Agrobacterium tumefaciens, a bacterium commonly found in soils. Usually the DNA from Agrobacterium that is transferred to the plants causes tumours in the recipient plant. To perform genetic engineering the bacterial genes are replaced with the genes of interest and infection of the plant with Agrobacterium does not result in tumour formation. This technology was first applied to eastern white pine and has proven to greatly facilitate and accelerate the production of transgenic conifers. We have successfully used this approach to introduce in white pine the coding region of the two AMPs provided by Demeter. Over 40 different lines were produced and are presently under molecular analysis. We already have identified several lines showing good expression of the introduced AMP coding sequences; whole plants will then be regenerated for subsequent confrontation with various fungal pests, including the white pine blister rust
(Cronartium ribicola). Different lines of poplars were also obtained with the Demeter peptides, and will be tested against Melampsora medusae and Septoria musiva, for example.

\section{Potential Environmental Impacts}

One last aspect of this research is to evaluate the potential environmental impact of the introduction of transgenic trees. First, AMP-encoding genes may affect the microbial ecology of the soil. We are currently doing tests to track changes in the microbial populations using molecular methods. To do so, a DNA extraction method from soil has been adapted and specific amplification (by PCR) of ribosomal sequences from eucaryotes and procaryotes has been performed. The DNA fragments obtained were sequenced as the initial step for identification of members of the microbial population. These data showed the presence of a wide variety of microorganisms and a similar approach will be used for determining changes in microbial populations after the introduction of transgenic poplar trees. The ability of the transgenic poplars to form mychorrizal interaction will also be tested. Secondly, DNA released into the soil by decaying tree leaves and roots could become available for incorporation by soil microbes. In order to make a full assessment of this risk, a similar method based on PCR amplification from DNA isolated from soil will allow us to determine the level of persistence of recombinant DNA from decaying transgenic trees in natural conditions.

\section{Outlook for the Future}

Genetic engineering of forest trees entails a major investment that can generate considerable benefits over the long term. This includes the increased protection that tree biotechnology offers against forest pests. In the present article, I have given a brief description of the advances achieved with poplar and pine in the context of fungal resistance. Similar programs are also in place for genetic engineering of trees for insect resistance. Another important program focuses on understanding, at the molecular genetic level, cone development in conifers to ultimately engineer inhibition of reproductive organs in order to avoid pollen and seed dispersion of the transgenic material. Clearly, the gains made so far have come about through riding on the coattails of technologies originally developed for important crop species. The research undertaken in plant molecular biology in the field of agriculture is not at all like that being done in forestry. Nevertheless, many genes involved in various physiological processes specific to trees are being studied at present. Knowledge of these processes is essential for effectively integrating biotechnologies into tree breeding work. 